\title{
Behaviour of the Broadest DIBs as a Function of $\mathrm{E}(\mathrm{B}-\mathrm{V})$
}

\author{
B. York ${ }^{1}$, P. Sonnentrucker ${ }^{1}$, L. M. Hobbs ${ }^{2}$, D. G. York ${ }^{2}$, \\ S. D. Friedman ${ }^{1}$, J. Dahlstrom ${ }^{3}$, D. E. Welty ${ }^{2}$, T. P. Snow ${ }^{4}$, \\ and B. L. Rachford ${ }^{5}$ \\ ${ }^{1}$ Space Telescope Science Institute, Baltimore, MD, USA \\ e-mail: york@stsci.edu \\ ${ }^{2}$ University of Chicago, Chicago, IL, USA \\ ${ }^{3}$ Carthage College, Kenosha, WI, USA \\ ${ }^{4}$ Center for Astrophysics and Space Astronomy, University of Colorado, Boulder, CO, USA \\ ${ }^{5}$ Embry-Riddle Aeronautical University, Prescott, AZ, USA
}

\begin{abstract}
We present the first results of a dedicated search for Diffuse Interstellar Bands that have profiles with FWHM $>6 \AA$. Broad DIBs have been noticed in past surveys using averages of multiple sight lines (e.g. Jenniskens \& Désert, 1994), but careful detection, measurement, and cataloguing for individual sight lines has not been done since the pioneering work of Herbig (1995). We have initiated an observing campaign using the Apache Point Observatory in order to obtain low-resolution spectra to search for such broad DIBs and monitor their behaviour from star to star. A first sample of 21 stars with $0.3<\mathrm{E}(\mathrm{B}-\mathrm{V})<3.3 \mathrm{mag}$, along with 15 matched low-reddening stars, were observed with the APO/DIS B400 $(\mathrm{R} \sim 450)$ and R300 $(\mathrm{R} \sim 1000)$ gratings to obtain spectra having $\mathrm{S} / \mathrm{N}>500$.
\end{abstract}

Keywords. ISM: lines and bands

\section{Data Reduction}

Observations were processed through an automated data reduction pipeline using python, including pyraf for a number of standard IRAF tasks. Once data were sorted by night, the following steps were taken for each night: creation of a bias-corrected super-flat, detection and elimination of saturated images $\dagger$, aperture location and size determination, optimal 1D spectral extraction, wavelength calibration, the creation of nightly spectral sums for each target, and extinction correction for each sightline. The nightly spectra were then continuum normalized using a tenth-order Legendre polynomial. Figure 1 shows the reduced spectra of nine of the stars observed with DIS after data reduction.

For stars with $\mathrm{O}$ and $\mathrm{B}$ spectral types, normalized stellar atmosphere models were produced for O and B stars using the TLUSTY code (Lanz \& Hubeny 2007). Each normalized DIS spectrum was then divided by its stellar model (Figure 2). The stellar model that best fit the DIS data toward HD 172028 is shown at the top in Figure 1.

\section{Measurement}

For each sight line, we constructed an optical depth (OD) spectrum using the continuumnormalized data. The DIB strength was measured by integrating the OD profile over a

$\dagger$ Saturated images are defined as those where more than half of the rows containing the target aperture contained $>100$ saturated pixels 

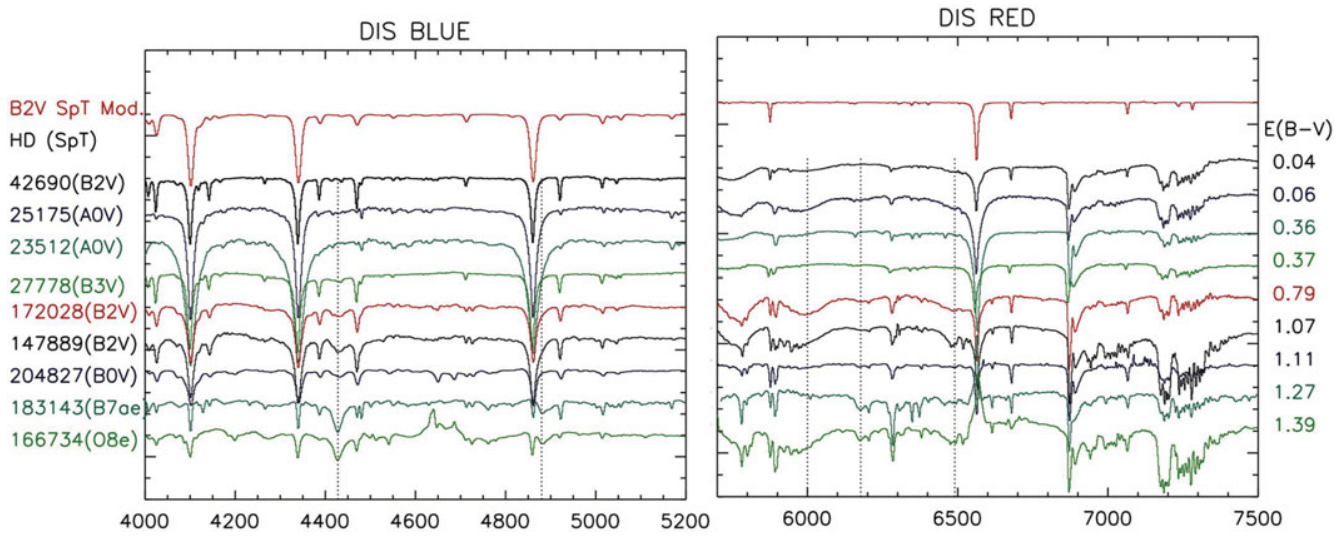

Figure 1. Normalized spectra obtained from APO/DIS blue (left) and red (right). Known broad DIBs $(4430,4480,6000,6177$, and $6500 \AA)$ are marked by vertical dotted lines. No other broad features are detected in the blue (left) panel. In the red panel, the $\sim 6000 \AA$ feature suffers telluric contamination below $5790 \AA$. The $\sim 6500 \AA$ feature is blended with stellar $\mathrm{H} \alpha$ whilst the $\sim 6177 \AA$ feature suffers mild blending with the DIS-unresolved 6196-6203 A DIB complex. The B2V stellar model for HD 172028 is plotted in red at the top for stellar line identification purposes. All spectra were displaced vertically for clarity. The star names and spectral types $(\mathrm{SpT})$ are listed on the left of each panel whilst $\mathrm{E}(\mathrm{B}-\mathrm{V})$ values are listed on the right.

Table 1. Comparison of DIB Strengths ${ }^{1}$

\begin{tabular}{|l|c|c|c|c|}
\hline DIB & \multicolumn{2}{|c|}{ HD 183143 } & \multicolumn{2}{c|}{ HD 204827 } \\
$\AA$ & Hobbs09 & DIS & Hobbs08 & DIS \\
& \multicolumn{2}{|c|}{ A } & \multicolumn{2}{|c|}{} \\
\hline 4430 & $5700 \pm 44$ & $3570 \pm 600^{3}$ & $1221 \pm 40$ & $645 \pm 90$ \\
5780 & $848 \pm 7$ & $995 \pm 250^{3}$ & $293 \pm 9$ & $441 \pm 150^{3}$ \\
5797 & $198 \pm 5$ & $181 \pm 80$ & $204 \pm 3$ & $243 \pm 80$ \\
$6196-6203$ & $459 \pm 7$ & $1260 \pm 500$ & $269 \pm 1$ & $213 \pm 40$ \\
6284 & $1884^{2}$ & $2526 \pm 200$ & $459^{2}$ & $877 \pm 70$ \\
6613 & $358 \pm 6$ & $365 \pm 30$ & $165 \pm 2$ & $132 \pm 40$ \\
\hline
\end{tabular}

Table 2. Strength of the $6177 \AA \mathrm{DIB}^{1}$

\begin{tabular}{|l|c|c|}
\hline STAR & $\begin{array}{c}\mathbf{E W}_{6177} \\
(\mathrm{~m} \AA)\end{array}$ & $\begin{array}{c}\mathbf{E}(\mathbf{B}-\mathbf{V}) \\
(\mathrm{mag})\end{array}$ \\
\hline HD 42690 & $<290(3 \sigma)$ & 0.04 \\
HD 25175 & $<420(3 \sigma)$ & 0.06 \\
HD 23512 & $<300(3 \sigma)$ & 0.36 \\
HD 27778 & $294 \pm 190$ & 0.37 \\
HD 172028 & $326 \pm 110$ & 0.79 \\
HD 147889 & $245 \pm 20$ & 1.07 \\
HD 204827 & $241 \pm 80$ & 1.11 \\
HD 183143 & $1657 \pm 150$ & 1.27 \\
HD 166734 & $1343 \pm 100$ & 1.39 \\
\hline
\end{tabular}

Notes:

${ }^{1}$ Note that the DIB strength was measured via apparent optical depth for the DIS spectra, and by equivalent width from fractional absorption for the ARCES spectrum.

${ }^{2}$ The APO/ARCES data are telluric corrected. The DIS data are not.

3 The 5780 DIB suffers from unresolved blending with other known DIBs causing the strength to be artificially larger with DIS compared to Hobbs et al. Due to unresolved stellar line blanketing the continuum placement and normalization of our DIS spectra results in a measurement of the $4430 \AA$ DIB that is systematically underestimated by at least $30 \%$ with respect to Hobbs et al. $(2008,2009)$.

fixed wavelength range for each DIB. The $1 \sigma$ error in DIB strength was estimated by shifting the best fit continuum by \pm the rms in the continuum and integrating the OD profile again. Table 1 compares APO/DIS measurements for HD 183143 and HD 204827 with APO/ARCES echelle data obtained by Hobbs et al. $(2008,2009)$.

\section{Results and Future Work}

The DIS data are consistent in most cases with Hobbs et al. $(2008,2009)$ for isolated and unblended DIBs. In other cases, the limitations inherent in continuum placement for broad DIBs in echelle spectra may have introduced significant errors into the ARCES equivalent widths, for DIBs specifically with FWHM $>6 \AA$ (Hobbs 2009; Searles et al. 2011). Our data confirm the presence of a broad DIB at $6177 \AA$, as shown in Table 2. The 


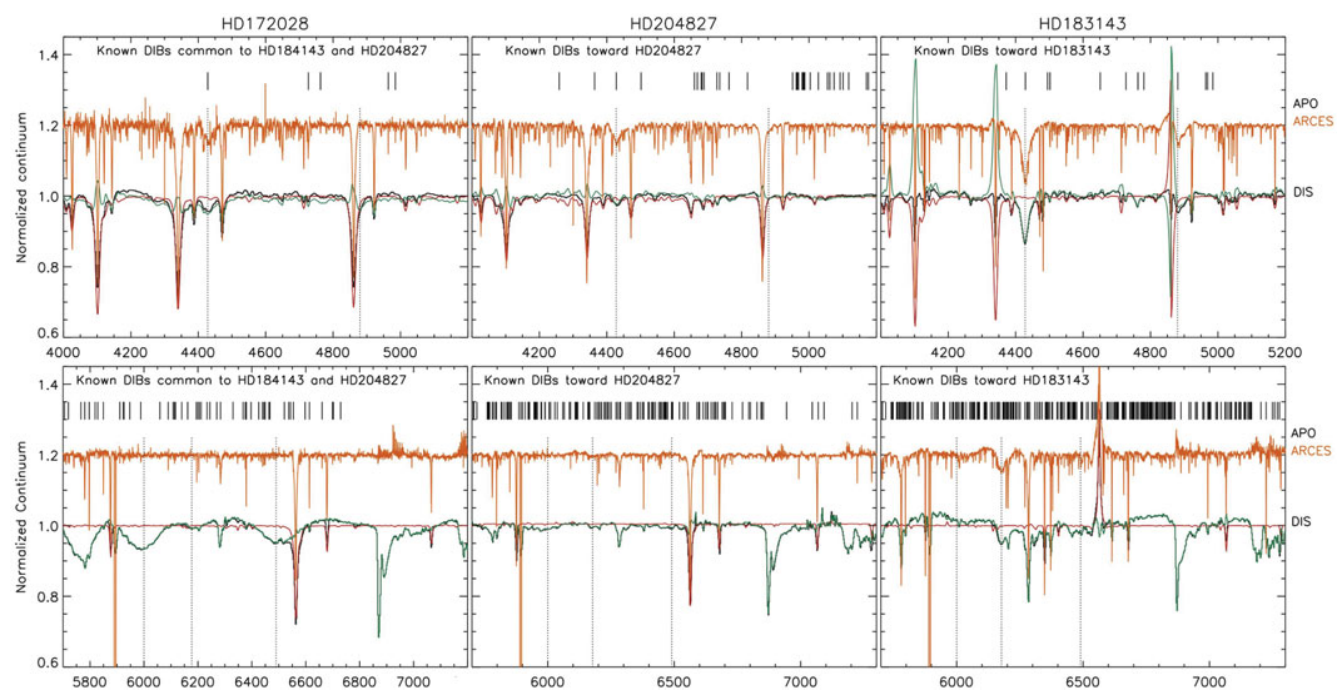

Figure 2. Normalized APO/DIS (black) and APO/ARCES echelle (orange) data from

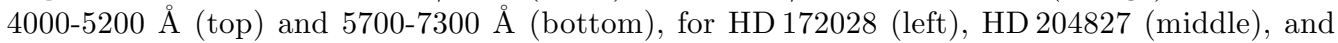
HD 183143 (right). The best stellar models are plotted in red over the DIS spectra, and the stellar-model-corrected spectra appear in green. All ARCES spectra are telluric-corrected whilst DIS spectra are not. In the red (>5700 $\AA$ ), a broad feature around $6177 \AA$ is detected toward all 3 sight lines. However, strong $6000 \AA$ and $6500 \AA$ features are only seen toward HD 172028 . In the blue $(<5200 \AA)$, the well-known $4430 \AA$ DIB is clearly seen in all cases. The feature around $4880 \AA$ reported in earlier atlases (e.g. Herbig 1995) is not detected toward these particular sight lines. No new broad features are detected in the blue. Note that our data are unreliable below $4000 \AA$ due to severe unresolved stellar line blanketing. The dotted vertical lines mark the position of the broad features seen in our data. The vertical tick marks indicate the position of the known DIBs listed in the atlases of HD 183143 (Hobbs et al. 2009) and HD 204827 (Hobbs et al. 2008). For HD 172028, the tick marks indicate the positions of those DIBs present toward both HD 183143 and HD 204827. The ARCES spectra were shifted vertically for clarity.

$6177 \AA$ DIB is weaker per unit reddening in sight lines containing $\mathrm{C}_{2}$ DIBs (HD 204827) than in HD 183143-type sight lines, as has been seen for other broad features (e.g. 6284, 4430). Additional broad features at $\sim 6000 \AA$ and $\sim 6500 \AA$ were also seen, but their DIB nature remains under investigation. The $4880 \AA$ DIB is present but blended with stellar $\mathrm{H} \beta$. No other previously reported broad features (see e.g. Herbig 1995 and references therein) were detected with our current survey.

\section{References}

Herbig, G. 1995, ARA\&A, 33, 19

Hobbs, L. M., York, D. G., Thorburn, J., Snow, T., Bishof, M., Friedman, S., McCall, B., Oka, T., Rachford, B., Sonnentrucker, P., \& Welty, D. 2008, APJ, 705, 32

Hobbs, L. M., York, D. G., Snow, T., Oka, T., Thorburn, J., Bishof, M., Friedman, S., McCall, B., Rachford, B., Sonnentrucker, P., \& Welty, D. 2009, APJ, 680, 1256

Jenniskens, P. \& Désert, F.-X. 1994, A\&ASS, 106, 39

Lanz, T. \& Hubeny, I. 2007, APJS, 169, 83

Searles, J., Destree, J., Snow, T., Salama, F., York, D. G., \& Dahlstrom, J. 2011, ApJ, 732, 50 